\title{
Edge Detection Comparison for License Plate Detection
}

\author{
Zuwena Musoromy, Dr. Soodamani Ramalingam \\ Department of Engineering and Technology \\ University of Hertfordshire \\ Hatfield, UK \\ \{z.musoromy,s.ramalingam\}@ herts.ac.uk
}

\author{
Nico Bekooy \\ CitySync Ltd. \\ Welwyn Garden City, UK \\ Nico.Bekooy@citysync.co.uk
}

\begin{abstract}
The detection of license plate region is the most important part of a vehicle's license plate recognition process followed by plate segmentation and optical character recognition. Edge detection is commonly used in license plate detection as a preprocessing technique. This paper compares the performance of the image enhancement filters when used in edge detection algorithms combined with connected component analysis to extract license plate region. The experimental comparison of Canny, Kirsch, Rothwell, Sobel, Laplace and SUSAN edge detectors on gray scale images shows that Canny yields high plate detection of $98.2 \%$ tested on 45,032 UK images containing license plates at $720 \times 288$ resolution captured under various illumination conditions. The average processing time of one image is $56.4 \mathrm{~ms}$.
\end{abstract}

Keywords: Edge detection, Connected component analysis (CCA), License plate (LP)

\section{INTRODUCTION}

Nowadays, vehicle License Plate Recognition (LPR) is becoming the key in Intelligent Transportation Systems (ITSs) with the main use being in Automatic Number Plate Recognition (ANPR) systems. This is an image-processing technology used to identify vehicles by their number plates. The systems are created depending on market area, character set, camera movement, imaging types, image processing algorithms, hardware platforms, processing power and cost. The ANPR systems usage is in car parking and traffic monitoring including highways, law enforcement, automotive, security, home monitoring and access control. There are various License Plate (LP) character sets including Australian [1], Chinese [2], Greek [3], Israel [4], Saudi [5], Spanish [6], Taiwan [7], Turkey [8], UK [9] and US [10] reported in the LPR literature. LPR is usually divided into three parts, which are license plate detection (the most important part), character segmentation and character recognition. LP detection is the process of finding the region or location in an image that contains the LP.

The aim of this work is to find algorithms that improve LP detection rate. In order to evaluate the LP detection performance, the testing data used is 45,032 UK LP images. This may seem to be a lot of images compared to other work performed so far as reported by the survey in [3]. The advantage of using 45,032 is that $1 \%$ in LP detection of this sample represents 450 LPs. This is a reasonable sample in situations such as busy roads like the highway where more cars use the road in any given day. This paper compares the performances of the edge detection algorithms on the gray scale images and their application in detecting license plates. Edge detection is an image processing technique. In gray scale images, an edge is defined by discontinuity in gray level values to form object boundaries. The object boundaries provide an advantage in object detection process from the image. It is well-known that the edges shape is determined by geometrical and optical properties of the object, image lighting conditions and noise level [11].

Image enhancement for edge detection compared in this work is performed in spatial domain. Three edges enhancement methods based on differentiation operators classified in [12] are investigated using gray-level image processing. These are first derivative gradient edge detectors (Kirsch and Sobel [28]), second derivative based on zero crossing (Laplacian [28]) and Gaussian edge detectors (Canny [18]). In addition, Rothwell [13] and SUSAN [14] edge detectors are also compared. The rest of this paper is organized as follows. Section (2) reviews previous work. Section (3) discusses edge detectors and CCA algorithms. Section (4) presents the LP detection algorithm. Section (5) presents experimental result of edge detections and LP detection obtained and finally Section (6) gives discussion and conclusion.

\section{REVIEW OF PREVIOUS WORK}

In this section, previous work carried out on gray scale images to find edges using enhancement filters (operators) and their application to LP detection is reviewed.

\section{A. Edge Detectors Comparison}

The performance of different edge enhancement operators is normally measured using subjective and objective methods. A subjective method is based on human visual analysis on the edge image while an objective one is based on signal to noise ratio as used in $[12,15]$. A classified and comparative study of edge detection algorithm is presented in [15] where Boie-Cox 
[16], Shen-Castan [17] and Canny [18] operators are better than the classic Laplacian of Gaussian (LOG), while LOG is better than Prewitt [27] and Sobel in case of noisy images. The comparison of edge detection and Hough transform techniques is reported in [19] where Canny, Rothwell, Black [20], Bezdek [21], Iverson-Zucker [22] and SUSAN operators are compared for satellite images. In this study, Canny performed best followed by Rothwell then SUSAN. Another comparison study is explained in [12]. In this paper it is shown that under noisy conditions, Shen-Castan [23], Canny, Marr-Hildreth [28], Kirsch, Sobel and Laplace demonstrate better performance, respectively.

\section{B. Licence Plate Detection with Edge Detectors}

This section reviews LP detection when edge enhancement algorithms are used. The detection of LP in [2] starts by enhancing the image, then applying Sobel operator to find vertical edges, followed by unwanted edges elimination and finally, the plate region is extracted. The publication in [4] starts LP finding by down sampling the input image, followed by Robert [28] operator to find vertical edges (left and right boundaries) and rank filter to get horizontal edges (top and bottom). In [5] and [26], the vertical Sobel edge detector is applied on the input image to give the resultant edge image, followed by unwanted vertical edges filtering and vertical edge matching compared to the known plate aspect ratio of a plate. Table I is generated as a summary of a review of edge detection enhancement filters for LP detection performed in this work. In the table, the results of LP detection success, algorithm used, algorithm performance in milliseconds (ms) and the sample size used for testing are shown.

The edge enhancement methods clearly show a good LP extraction rate and a fast extraction time is reported in [24]. The authors also reported that the main shortcomings of the methods chosen being bad quality input image during the image capture stage and unclear edges extraction which leads to failure to detect LP. It is normally expected that the LP detection goal is always to achieve a real-time result and high recognition rate. However, the former depends very much on the processor and algorithms used. The review shows encouraging results in LP detection using edge enhancement operators. This work will further investigate the performance of Canny, Kirsch, Laplace, Rothwell, Sobel and SUSAN operators on gray scale images.

\section{EDGE DETECTORS AND CONNECTED COMPONENT ANALYSIS}

This section provides Canny, Kirsch, Laplace, Rothwell, Sobel and SUSAN operators with CCA definitions.

The Sobel operator is regarded as a classical edge detector. It convolves the image using small integer valued filter in horizontal and vertical directions using a $3 \times 3$ mask for each direction. The mask is applied over the whole image, processing a square of pixels at a time. This gives emphasis to regions of high frequency that corresponds to edges as shown in Fig. 2. In other words, it is a two dimensional spatial gradient measurement on an image [28]. This operator is relatively inexpensive due to small integer value and therefore it is very popular in image processing as shown in the application of LP detection [1], [2], [5], [25] and [26].

Kirsch is similar to Sobel as explained in [28]. This operator introduced a more practical way to avoid mathematical operations needed to calculate the square root of the sum of the squares needed by the Sobel. This method applies the eight orientations of the derivative kernel and keeps the maximum value. It requires only integer multiplication and comparisons [28].

The Laplace algorithm works the exact same way as the Sobel. The only difference is that Laplace uses one mask for the second derivative in both the vertical and horizontal directions as in [23] and [28]. However, because the mask is approximating a second derivative measurement on the image, it is very sensitive to noise as shown in Fig. 3. The LoG is introduced to reduce the noise. In LoG the image is firstly smoothed with a Gaussian filter then Laplace operator is applied [28].

The Canny edge detector is classified as a Gaussian edge detector according to [12]. This is because the filter can be approximated by first-order derivatives of Gaussians. It can be explained by a number of steps. Firstly, the image is smoothed to eliminate any noise; it then finds the image gradients to emphasize regions with high spatial derivatives. These regions are tracked and any pixel within the region that is not at the maximum is suppressed. At this point hysteresis is introduced to track along the rest of the pixels that have not been suppressed. Two thresholds are set for hysteresis, which are compared to the gradient magnitude to find edge and non edge [18]. The edges are illustrated in Fig. 4.

The Rothwell algorithm for edge detection is designed to recover reliable topological descriptions as in [13]. In this algorithm the image is smoothed to account for image quantization, curved boundaries are fitted to provide reliable geometric descriptions of edges chain and finally the scene topology is recovered to detect object boundaries as shown in Fig. 5.

TABLE I. LITERATURE REVIEW LICENCE PLATE DETECTION RESULTS

\begin{tabular}{ccccc}
\hline Ref. & $\begin{array}{c}\text { Edge } \\
\text { Detection } \\
\text { Algorithm }\end{array}$ & $\begin{array}{c}\text { Sample } \\
\text { Size }\end{array}$ & $\begin{array}{c}\text { Success } \\
\%\end{array}$ & $\begin{array}{c}\text { Performance } \\
(\mathbf{m s})\end{array}$ \\
\hline$[1]$ & Sobel & $\begin{array}{c}\text { Not } \\
\text { reported } \\
\text { Not } \\
\text { [2] }\end{array}$ & $\begin{array}{c}\text { Not } \\
\text { reported } \\
97.0\end{array}$ & $\begin{array}{c}\text { Not } \\
\text { reported } \\
500\end{array}$ \\
{$[4]$} & $\begin{array}{c}\text { Sobel vertical } \\
\text { Robert and } \\
\text { Rank }\end{array}$ & 1000 & 96.3 & 227 \\
{$[5]$} & $\begin{array}{c}\text { Sobel vertical } \\
\text { Smoothing } \\
\text { Filter and } \\
\text { Edge } \\
\text { Mapping }\end{array}$ & 610 & 96.2 & $\begin{array}{c}\text { Not } \\
\text { reported } \\
100\end{array}$ \\
{$[25]$} & Sobel & 104 & 96.0 & \\
{$[26]$} & Sobel & 710 & 96.05 & $\begin{array}{c}\text { Not } \\
\text { reported } \\
\text { Not } \\
\text { reported }\end{array}$ \\
\hline
\end{tabular}


SUSAN (Smallest Univalue Segment Assimilating Nucleus) principle is complex. However, it is divided in steps and designed to perform edge detection, corner detection and structure-preserving image noise reduction [14]. In the edge detection part, a circular mask is placed over the pixel to be tested known as the nucleus and every pixel is then compared to it using a smoothed 'Top-Hat' function. A positive score is only returned if the area is small enough (SUSAN) and this is found using non-maximal suppression. The edges are shown in Fig. 6. Corner detection is achieved by adding two more steps. The first one is to find the centroid of the SUSAN, which is far from the nucleus. The final step condition for corner detection is that all points on the line from nucleus through the centroid outside of the edge of the mask are in SUSAN. This is explained in detail in [14].

CCA is a well-known technique in image processing. It operates by scanning an image and labels its pixels into components in order to identify connected pixel regions that share similar pixel intensity value. If the intensity is given as $Z$, for binary images

$$
Z=\{1\} .
$$

The intensity for gray scale image with 256 levels is given as,

$$
Z=\{0,2,3 . ., 255\} \text {. }
$$

CCA labeling works on binary or gray scale images and different measures of connectivity either four-connected or eight-connected may be used. The four-connected means that the pixel coordinates $\left(\mathrm{X}_{\mathrm{Z}}, \mathrm{Y}_{\mathrm{Z}}\right)$ are neighbors and touches pixels $\left(\mathrm{X}_{\mathrm{Z}} \pm 1, \mathrm{Y}_{\mathrm{Z}}\right)$ and $\left(\mathrm{X}_{\mathrm{Z}}, \mathrm{Y}_{\mathrm{Z}} \pm 1\right)$ edges in horizontal and vertical direction.

The eight-connected means that the pixel coordinates $\left(X_{Z}\right.$, $\left.\mathrm{Y}_{\mathrm{Z}}\right)$ are neighbors and touches pixels $\left(\mathrm{X}_{\mathrm{Z}} \pm 1, \mathrm{Y}_{\mathrm{Z}} \pm 1\right)$ and $\left(\mathrm{X}_{\mathrm{Z}}\right.$ $\pm 1, Y_{Z} \pm 1$ ) edges in diagonal, horizontal and vertical direction. CCA in binary images for LP extraction is reported in [29] and [30].

\section{LICENSE PLATE DETECTION ALGORITHM}

The plate detection algorithm used here is divided into four parts. These are input image normalization, edges enhancement using filters, edges finding and linking to rectangles (CCA) and plate candidate finding. In this study, connected component is performed on gray scale images and four-connected CCA is used. This algorithm is based on gray scale image processing. The algorithm is shown in Fig 7.

\section{A. Normalization}

The quality of images captured differs significantly depending on location and lighting conditions. This results in variable intensity levels even on flat surfaces. The majority of the image processing is performed on the edges therefore the selection of tolerable threshold is required in order to create a correct number of edges. This is the difference between maximum and minimum gray scale values.

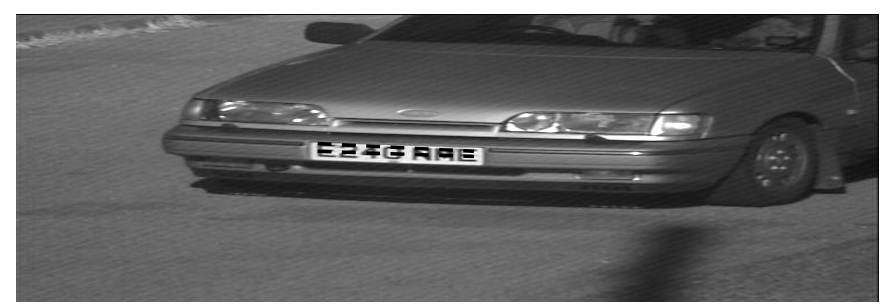

Figure 1. Input test image

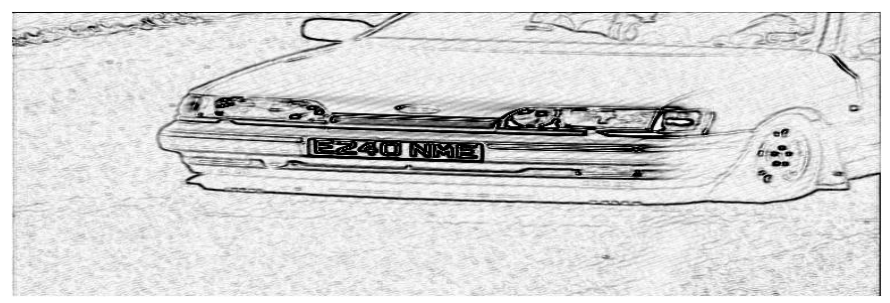

Figure 2. Sobel edges image

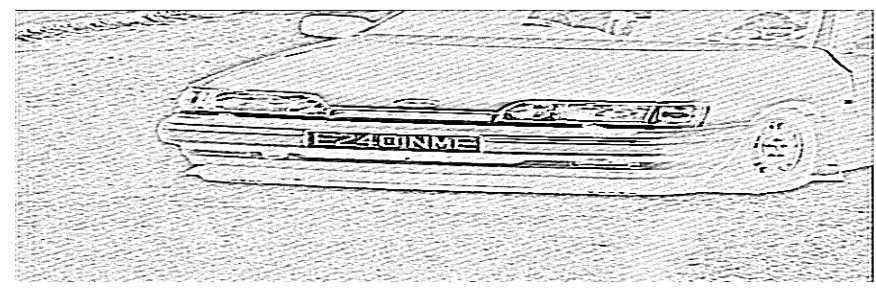

Figure 3. Laplace edges image

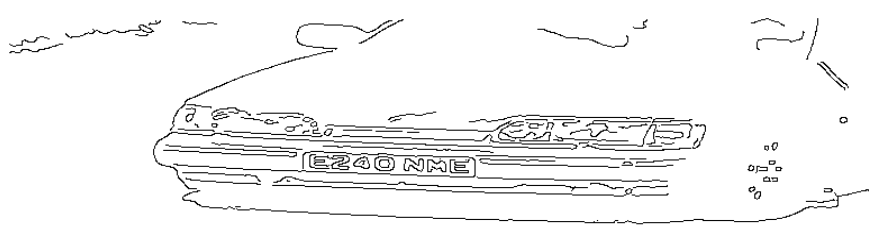

Figure 4. Canny edges image

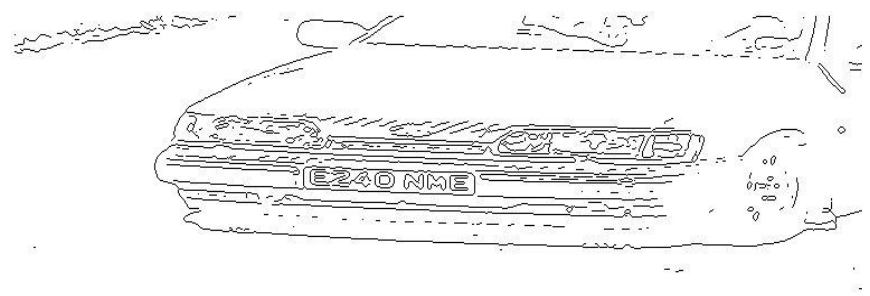

Figure 5. Rothwell edges image

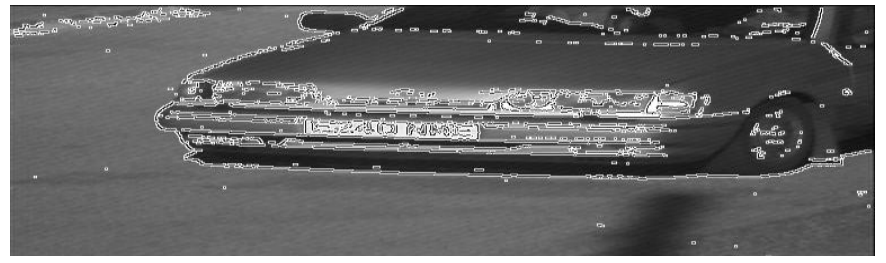

Figure 6. SUSAN edges image 
During image acquisition stage, the aim is to obtain images that are not too dark or too bright. The former will mean a very low threshold will needed to be set and therefore detail is lost within the plate whereas the latter will mean that not only will a high threshold will be needed but it carries with it the danger that the characters on the plates will be 'bleached out'. Fig. 2 shows original image while Fig 8 shows a high response to noise when normalization threshold is set low. This will lead to more false candidate rectangles (in white) being detected. The presence of false edges (noises) will slow down the process, and hence the appropriate threshold level is selected before calculating the edges present in the picture. In theory, good quality plate images contain less than $1 \%$ edges per image. In general, dark images have few edges and the difference between black and white (plate contrast) will be low while bright images have high contrast and therefore the threshold value is set high to minimize the edges.

\section{B. Edges Enhancement using Filters}

After normalization, Sobel, Kirsch, Laplace, Canny, Rothwell and SUSAN filters are applied to enhance edges and their performance is investigated. Edges are enhanced using various thresholds as shown in Fig. 2 to 6 . The best result is then added to the original image for processing. At this point some of the unwanted edges (noises) have been reduced and the threshold is passed on to the next step. Fig. 9 and Fig. 10 show horizontal and vertical edges.

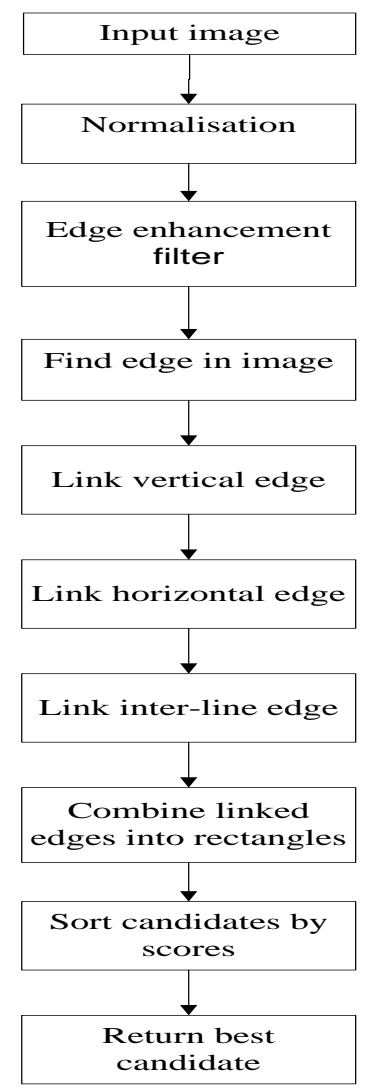

Figure 7. License Plate Detection Algorithm

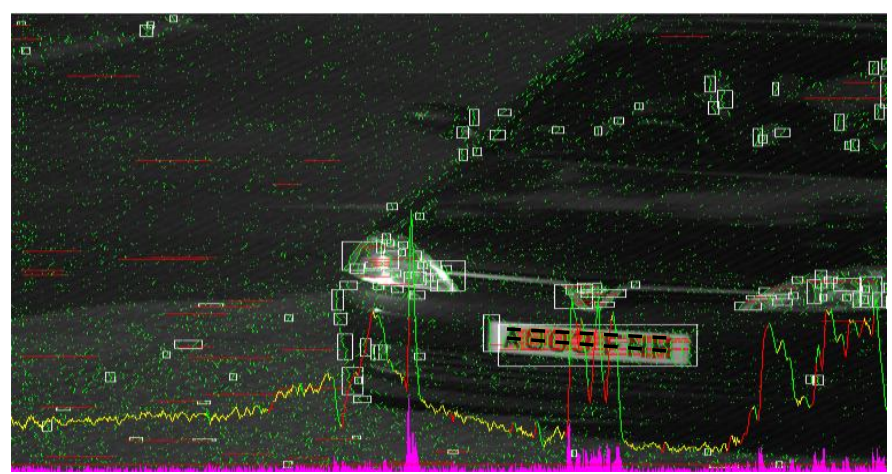

Figure 8. Noisy response to low normalisation threshold with histogram showing intensity variation on the plate image

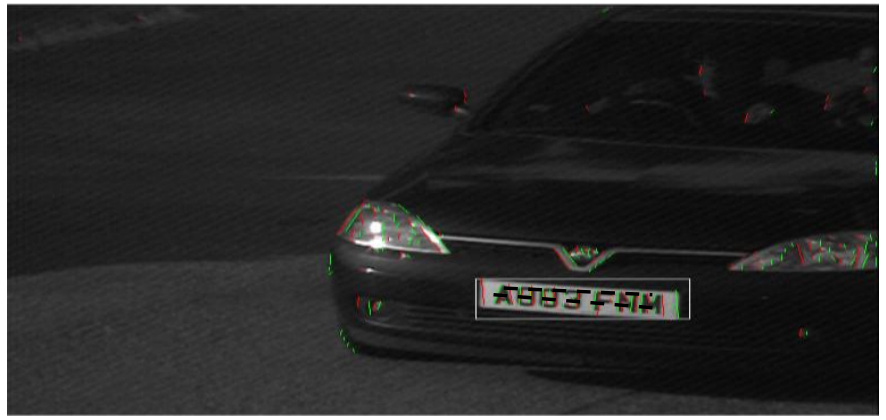

Figure 9. Vertical edges on original image

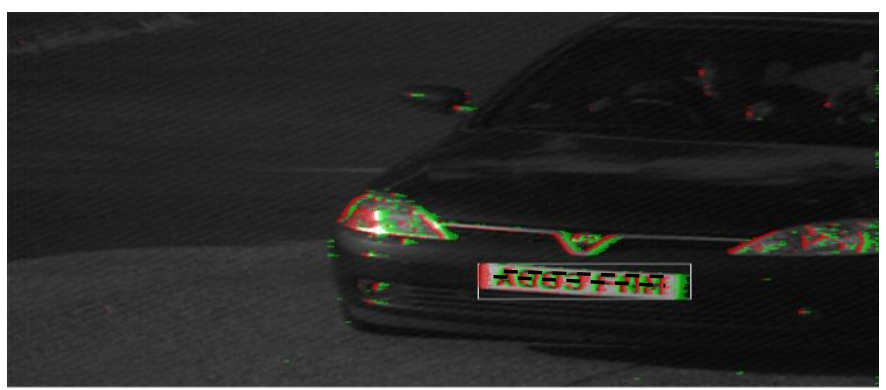

Figure 10. Horizontal edges on original

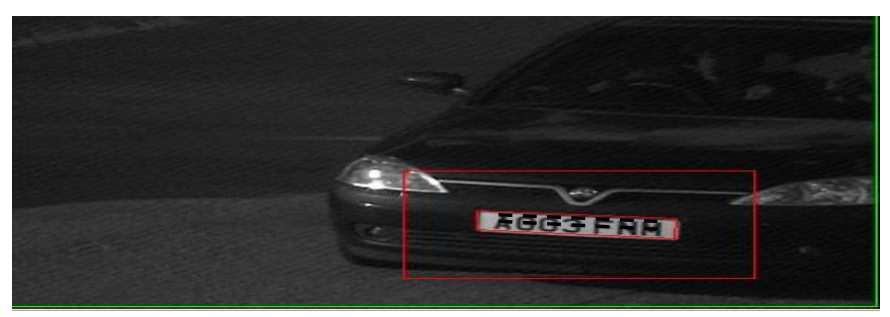

Figure 11. Licence plate detected

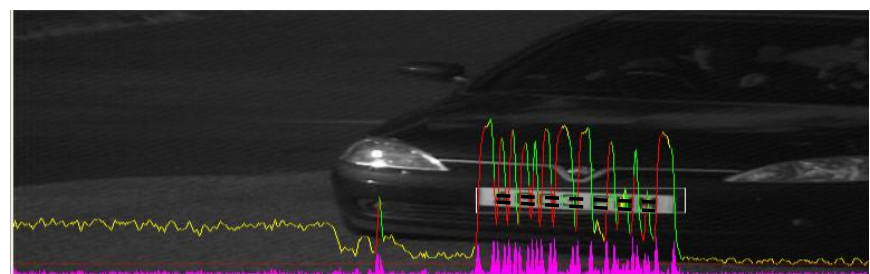

Figure 12. Histogram showing intensity variation on the plate image 


\section{Edges Finding}

The next step is to scan the image and a list of rising and falling edges is found using pixel variation using the original gray scale image with edges threshold provided from the previous step. The plate properties are noted where the text is usually in one color, and often on a plain background, therefore there should be a high number of edges on the plates (typically 100-2000 edges) compared to other parts of the image. This is also illustrated in Fig. 12 where the image histogram is plotted.

\section{Edges Linking and Plate Candidate Finding}

This is the final step. The horizontal and vertical edges are linked and bounding rectangles are calculated and compared, one for each potential plate within the image. This procedure results in false identification of candidate rectangles as shown in Fig. 8, which are eliminated by comparing with the plate known resolution of 140x14 (about 2000 pixels) to obtain the candidate rectangle as shown in Fig. 11.

\section{EXPERIMENTAL RESULTS}

Two types of experiments are carried out. The first one is to extract plate without edges enhancement filters and the second one is to apply filters and compare their performance. The processor used is Intel $2.4 \mathrm{GHz}, 3 \mathrm{~GB}$ of RAM PC using C language under Visual Studio 2008. The algorithm is tested using 45,032 UK images containing number plates. The images are a mixture of color, gray scale and Infra Red. In addition, the algorithm shows $99.8 \%$ plate detection rate when tested on the Greek online database provided by [3].

In the Canny algorithm, the parameters are set as follows; Sigma $=1.30$, lower threshold $=0.50$ and higher threshold high $=0.90$. In Rothwell, the parameters are set as Sigma $=1.0$, lower threshold $=12.0$ and higher threshold $=0.90$. In SUSAN the fast edge mode is selected with the threshold parameter set at 20. In Sobel and Kirsch the classical horizontal and vertical $3 \times 3$ masks were used. In Laplace $5 \times 5$ mask is used. These parameters were selected by testing a mixture of images (noisy and less noisy).

Table II presents the overall results. Plate Extraction Time (PET) is the time for plate detection. The results shows that the time to process a single image containing plate is faster than the time it takes to process an image without a plate. This is because the algorithm scans through the whole image again before moving to the next image when there is no plate to be found.

\section{DISCUSSION AND CONCLUSION}

Canny performs best compared to the algorithm without enhancement filters with an improvement of $2.5 \%$ however; it is slow and may struggle to achieve a real-time requirement (25 frames per second). The fastest time is when no filters are applied. This is expected as there is less computations taking place. This algorithm is performing at real-time.
TABLE II. EXPERIMENTAL LICENSE PLATE DETECTION RESULTS

\begin{tabular}{cllll}
\hline $\begin{array}{c}\text { Algorith } \\
\mathbf{m}\end{array}$ & $\begin{array}{l}\text { 1 image } \\
\text { PET } \\
(\mathbf{m s})\end{array}$ & $\begin{array}{l}\text { No plate } \\
\mathbf{1} \text { image PET } \\
(\mathbf{m s})\end{array}$ & $\begin{array}{l}\mathbf{4 5 , 0 3 2} \text { images } \\
\text { PET } \\
(\mathbf{m s})\end{array}$ & $\begin{array}{l}\text { Success } \\
(\%)\end{array}$ \\
\hline Laplace & 6.6 & 14.4 & 64.9 & 90.8 \\
Sobel & 6.2 & 14.2 & 62.7 & 91.4 \\
Kirsch & 5.9 & 13.6 & 54.5 & 91.4 \\
Canny & 6.8 & 15.2 & 56.4 & 98.2 \\
Rothwell & 10.4 & 21.0 & 87.9 & 97.4 \\
SUSAN & 5.5 & 12.4 & 39.2 & 96.7 \\
No filters & 2.1 & 5.6 & 18.8 & \\
& & & &
\end{tabular}

The drawback of not enhancing the images in this algorithm when using this particular testing database is that lower LP detection rate is observed compared to Canny, Rothwell and SUSAN. SUSAN is also fast but shows less plate detection rate compared to Canny.

Overall, in terms of plate detection rate, the descending order is Canny, Rothwell, SUSAN, non enhanced algorithm, Kirsch, Sobel and Laplace. In terms of performance time, the descending order is non enhanced algorithm comes first followed by SUSAN, Kirsch, Canny, Sobel, Laplace and Rothwell. The summary is shown in Table II. The improvement of LP detection when using Canny, Rothwell and SUSAN is due to clear edges (less noisy) results provided by the operators. It is noted that the algorithms return $2 \%$ false positives (falsely identified) plates. The undetected plates are due to bad plates and complex lighting conditions when the plate is too dark, too bright or damaged while the false positives are due to the CCA high response to edges.

To conclude, the results shows that edge enhancement filters with less noises can be used to improve LP detection when combined with CCA on gray scale images. However, a good balance is needed in terms of speed and recognition rate to determine the choice of the best algorithm. Future work will focus on finding and further improving edge detector for license plate detection with the aim of achieving higher and accurate LP detection in real-time. With speed in mind, fast processors will also be investigated.

\section{ACKNOWLEDGMENT}

Acknowledgment is passed to Carol McStravick for her invaluable advice, editing and support, Prof. Aladdin Ariyaeenia, Dr. Faycal Bensaali, Dr. Georgios Pissanidis, Prof. Reza Sotudeh and Bob Andrews for their support. 


\section{REFERENCES}

[1] S. Gendy, C.L Smith, S. Lachowicz, "Automatic car registration plate recognition using fast Hough transform," Security Technology Proceedings. The Institute of Electrical and Electronics Engineers 31st Annual 1997 International Carnahan Conference on , vol., no., pp.209$218,15-17,1997$.

[2] D. Zheng, Y. Zhao, and J. Wang, "An efficient method of license plate location," Pattern Recognit. Lett., vol. 26, no. 15, pp. 2431-2438, 2005.

[3] C. E. Anagnostopoulos, I. E. Anagnostopoulos, I. D.Psoroulas, V. Loumos, and E. Kayafas, "License platerecognition from still images and video sequences: Asurvey," IEEE ransactions on Intelligent Transportation System, vol. 9, no. 3, pp. 377-391, 2008.

[4] Shapiro, Vladimir, Gluhchev, Georgi, Dimov, Dimo, "Towards a Multinational Car License Plate Recognition System," MVA(17), No. 3, pp. 173-183, 2006.

[5] Muhammad Sarfraz, Mohammed Jameel Ahmed, Syed A. Ghazi, "Saudi Arabian License Plate Recognition System," gmag, pp.36, 2003 International Conference on Geometric Modeling andGraphics (GMAG'03), 2003.

[6] J. Cano and J. C. Perez-Cortes, "Vehicle license plate segmentation in natural images," in Lecture Notes on Computer Science, vol. 2652, F. J. Perales et al., Eds. New York: Springer-Verlag, pp. 142-149, 2003.

[7] S. Z. Wang and H. M. Lee, "Detection and recognition of license plate characters with different appearences," in Proc. Conf. Intell. Transp. Syst., vol. 2, pp. 979-984, 2003.

[8] F. Kahraman, B. Kurt, and M. Gökmen, "License plate character segmentation based on the gabor transform and vector quantization," in Lecture Notes on Computer Science, vol. 2869, A. Yaziciand C. Sener, Eds. New York: Springer-Verlag, pp. 381-388, 2003.

[9] Barroso, J.; Dagless, E.L.; Rafael, A.; Bulas-Cruz, J., "Number plate reading using computer vision," Industrial Electronics, 1997. ISIE '97., Proceedings of the IEEE International Symposium on , vol., no., pp.761766 vol.3, 7-11 Jul 1997.

[10] L. Dlagnekovin License Plate Detection Using AdaBoost. La Jolla: Comput. Sci. Eng. Dept., Univ. California San Diego, Mar. 2004.[Online]. Available at: http://www.cse.ucsd.edu/classes/fa04/cse252c/projects/louka.pdf, Accessed 25th March 2010.

[11] H.Chidiac, D.Ziou, "Classification of Image Edges",Vision Interface'99, Troise-Rivieres, Canada, 1999. pp. 17-24.

[12] Sharifi, M.; Fathy, M.; Mahmoudi, M.T.; " A classified and comparative study of edge detection algorithms", International Conference on Information Technology: Coding and Computing, Proceedings, Page(s): 117 - 120, 8-10 April 2002.

[13] Rothwell, Ch., J. Mundy, B. Hoffman and V. Nguyen, 1994.Driving Vision by Topology. TR-2444 - Programme 4, INRIA, pp. 1-29.

[14] Smith, S. M. and J. M. Brady, 1997. SUSAN- A New Approach to Low Level Image Processing. International Journal of Computer Vision, 23(1), pp. 45-78.
[15] Roushdy M., "Comparative Study of Edge detection Algorithms Applying on the Grayscale Noisy Image Using Morphological filter", ICGST, Intenational Journal of Graphics, Vision, and Image Processing GVIP, Vol. 6, Issue 4, pp. 17-23, , Dec. 2006.

[16] Cox, I.J.; Boie, R.A.; Wallach, D.A.; "Line recognition", Pattern Recognition Proceedings., 10th International Conference on Volume i, Page(s):639 - 645 vol.1, June 1990

[17] Castan, S.; Zhao, J. and Shen, J." New edge detection methods based on exponential filter",Pattern Recognition, Proceedings 10thInternational Conference on Volume i, Issue 16-Page(s):709 - 711, vol.1, Jun 1990

[18] Canny, J. F., A computational approach to edge detection. IEEE Transactions. On Pattern Analysis and MachineIntelligence, 8, 1986, pp. 679-714.

[19] Argialas, D. P. and O. D. Mavrantza, "Comparison Of Edge Detection And Hough Transform Te hniques In Extraction Of Geologic Features". In Proceedings of the XX ISPRS Congress of the International Society of Photogrammetry and Remote Sensing, 12-23 July 2004, Istanbul, Turkey, pp. 790-795, Vol. IAPRS-XXXV, ISSN 1682-1750.

[20] Black, M., G. Sapiro, D. Marimont and D. Heeger, "Robust Anisotropic Diffusion". IEEE Transactions on Image Processing, 7, pp. 421-432, 1998.

[21] Bezdek, J. C., R. Chandrasekhan and Y. Attikiouzel, "A geometric approach to edge detection". IEEE Transactions. On Fuzzy Systems, 6, pp. 52-75, 1998.

[22] Iverson, L. A. and S. W. Zucker, Logical / linear operators for image curves. IEEE Transactions on Pattern Analysis and Machine Intelligence, 17(10), pp. 982-996, 1995

[23] Albovik,"Handbook of Image and Video Processing", Academic Press, 2000.

[24] H. Bai, J. Zhu, and C. Liu, "A fast license plate extraction method on complex background," Intelligent Transportation Systems, 2003.Proceedings. 2003 IEEE, vol. 2, pp. 985-987, 2003.

[25] SherrZheng Wang and HsMian Lee, "Detection and Recognition of License Plate Characters with Different Appearences", in proc. Conf. Intelligent Transportation Systems, vol. 2, 2003, pp. 979 - 984.

[26] Ahmed, M.J.; Sarfraz, M.; Zidouri, A.; Al-Khatib, W.G., "License plate recognition system," Electronics, Circuits and Systems, 2003. ICECS 2003. Proceedings of the 2003 10th IEEE International Conference on, vol.2, no., pp. 898-901 Vol.2, 2003.

[27] Raman Maini and J. S. Sohal, "Performance Evaluation of Prewitt Edge Detector for noisy Images", GVIP Journal, Vol. 6, Issue 3, December 2006.

[28] Russ, "The image processing handbook", CRC Press, 2002

[29] D. Llorens, A. Marzal, V. Palazon, and J. M. Vilar, "Car license plates extraction and recognition based on connected components analysis and HMM decoding," in Lecture Notes on ComputerScience, vol. 3522, J. S. Marques et al., Eds. New York: Springer-Verlag, pp. 571-578, 2005.

[30] P. Wu, H.-H. Chen, R.-J. Wu, and D.-F. Shen, "License plate extractionin low resolution video," in Proc. 18th ICPR, Hong Kong, 2006, vol. 1, pp. 824-827. 